Article

\title{
Performance-Enhanced Activated Carbon Electrodes for Supercapacitors Combining Both Graphene-Modified Current Collectors and Graphene Conductive Additive
}

\author{
Rubing Wang ${ }^{1,2}$, Yuting Qian ${ }^{1}$, Weiwei Li ${ }^{1,3}$, Shoupu Zhu ${ }^{1}$, Fengkui Liu ${ }^{1}$, Yufen Guo ${ }^{1,3}$, \\ Mingliang Chen ${ }^{1}$, Qi $\mathrm{Li}^{1, *}$ and Liwei Liu ${ }^{1,3, *}$ \\ 1 Key Laboratory of Nanodevices and Applications, Suzhou Institute of Nano-Tech and Nano-Bionics, \\ Chinese Academy of Sciences, Suzhou 215123, China; rbwang2013@sinano.ac.cn (R.W.); \\ ytqian2016@sinano.ac.cn (Y.Q.); wwli2009@sinano.ac.cn (W.L.); spzhu2017@sinano.ac.cn (S.Z.); \\ fkliu2015@sinano.ac.cn (F.L.); yfguo2009@sinano.ac.cn (Y.G.); mlchen2011@sinano.ac.cn (M.C.) \\ 2 School of Nano Technology and Nano Bionics, University of Science and Technology of China, \\ Hefei 230026, China \\ 3 SZGraphene Nanotechnology Co., Ltd., Suzhou 215123, China \\ * Correspondence: qli2013@sinano.ac.cn (Q.L.); 1wliu2007@sinano.ac.cn (L.L.); Tel.: +86-512-62872734 (L.L.)
}

Received: 17 April 2018; Accepted: 9 May 2018; Published: 15 May 2018

\begin{abstract}
Graphene has been widely used in the active material, conductive agent, binder or current collector for supercapacitors, due to its large specific surface area, high conductivity, and electron mobility. However, works simultaneously employing graphene as conductive agent and current collector were rarely reported. Here, we report improved activated carbon (AC) electrodes (AC@G@NiF/G) simultaneously combining chemical vapor deposition (CVD) graphene-modified nickel foams (NiF/Gs) current collectors and high quality few-layer graphene conductive additive instead of carbon black (CB). The synergistic effect of NiF/Gs and graphene additive makes the performances of AC@G@NiF/G electrodes superior to those of electrodes with CB or with nickel foam current collectors. The performances of AC@G@NiF/G electrodes show that for the few-layer graphene addition exists an optimum value around $5 \mathrm{wt} \%$, rather than a larger addition of graphene, works out better. A symmetric supercapacitor assembled by AC@G@NiF/G electrodes exhibits excellent cycling stability. We attribute improved performances to graphene-enhanced conductivity of electrode materials and $\mathrm{NiF} / \mathrm{Gs}$ with 3D graphene conductive network and lower oxidation, largely improving the electrical contact between active materials and current collectors.
\end{abstract}

Keywords: activated carbon; supercapacitor; electrodes; graphene; nickel foam; current collector; conductive additive; electrochemical properties

\section{Introduction}

With the increasing requirement of energy storage devices in the modern technology society, supercapacitors have drawn great attention due to the higher power density, quicker charge and discharge rate, and longer cycle life compared with traditional lithium-ion batteries [1-4]. According to the different charge storage modes, supercapacitors can be divided into two kinds, the pseudocapacitor derived from the reversible redox reaction of active electrode material and electrical double-layer capacitor (EDLC) depending on electrostatic ion attraction and accumulation on the electrode/electrolyte interface [5-7].

At present, the relatively low energy density is still a key bottleneck of supercapacitors. The formula $\mathrm{E}=\frac{1}{2} \mathrm{CV}^{2}$, where $\mathrm{E}$ is the energy density, $\mathrm{C}$ is the mass specific capacitance, and $\mathrm{V}$ 
is the potential range, illustrates that energy density is closely related to the specific capacitance and potential range [8-11]. As a result, improving specific capacitance or potential range is the fundamental approach to achieve higher energy density. Now, most researches concentrate on selecting appropriate active materials and electrolytes to get higher specific capacitances and broader working voltages. Carbon materials (graphene, carbon nanotube, AC et al.) with high conductivity, nanostructure transition metal oxide $\left(\mathrm{MnO}_{2}, \mathrm{Co}_{3} \mathrm{O}_{4}, \mathrm{Ni}_{X} \mathrm{Mn}_{1-X} \mathrm{O}\right.$ et al.) with high capacitance and their composites are the most famous active materials being studied now [12-17]. Furthermore, asymmetric supercapacitors have also been fabricated to obtain higher energy densities through broadening working voltages $[18,19]$. However, there are many other factors on equivalent series resistance (ESR) easily to be neglected, such as current collectors and conductive additives, which often influence performance enhancement by decreasing the resistances of the active materials, current collectors, the contact resistances between them, and so on [20-23].

Current collector, as an essential part of a supercapacitor, plays an important role in collecting current and supporting electrode materials. The conductivity and adhesion with active materials of a current collector can directly influence the specific capacitance [24-27]. Nickel is one of the most popular current collectors of supercapacitors owing to its good conductivity, mechanical properties, stability in aqueous alkali and strong adhesions with active materials [28-30]. To further improve the above properties is still the main research orientation in modifying nickel current collectors. Many works about modifying nickel current collectors by chemical or electrochemical etches have been published to increase the surface roughness in order to strengthen the adhesion with active materials [31,32]. In addition, methods like jet electrodepositing were reported to prepare a multi-pore nickel to increase the surface roughness [33]. However, these methods are always involved in the usage of alkali or acid solutions, and even relatively complex fabrication processes, which are environmentally unfriendly, lack safety, and are difficult in industry promotion. And then a kind of modified nickel foam $(\mathrm{NiF})$ current collectors with intrinsic high specific surface area, covered by few layer graphene through chemical vapor deposition (CVD) has been reported [21,34]. These graphene-modified NiFs can not only increase the conductivity of the current collectors but also strengthen the combination between active materials and current collectors, markedly improving the electrical contacts of active materials and current collector. In addition, the existence of the CVD graphene layers protects the NiFs from the erosion of oxygen and water in the air, which has been rarely published.

Moreover, conductive additives also are of great importance in an operational electrode. The most common conductive additive is carbon black (CB) [35]. The additive amounts of CB are always up to $10 \mathrm{wt} \%$ of the total electrode weight, even more in some poor conductivity systems, which makes the proportion of active material reduced, as well as the overall specific capacitance. Efficient conductive additives are needed to achieve high performance electrodes with lower additive amounts. Highly conductive graphene is a potential candidate for conductive additives because of its well-known excellent properties, such as high conductivity, specific surface area, light weight, and so on [36,37].

Here, activated carbon (AC) electrodes were prepared with CVD graphene-modified nickel foams (NiF/Gs) as current collectors and high quality few-layer graphene as conductive additive (AC@G@NiF/G). On one hand, few-layer graphene as conductive additive directly improves the conductivity of the electrodes. On the other hand, CVD graphene on NiFs not only increases the conductivity and stability of the current collector, but also as a buffer layer to strengthen the adhesion to active material, providing a highly effective electrical contact for fast charge transfer from AC active material to current collectors with the underlying 3D graphene network [21]. Due to the synergistic effect of $\mathrm{NiF} / \mathrm{G}$ current collectors and few-layer graphene conductive additive, the electrochemical properties of as-prepared AC@G@NiF/G electrodes are higher than those of AC electrodes with $\mathrm{CB}$ conductive additive (AC@CB@NiF/G) and common NiF current collectors free of graphene (AC@G@NiF). Just at low weight of graphene conductive additive of 5 wt \%, the AC@G@NiF/G (denoted as AC@G@NiF/G-5) electrode shows highest electrochemical properties 
(specific capacitance $123.6 \mathrm{~F} / \mathrm{g}$ and energy density $17.2 \mathrm{Wh} / \mathrm{kg}$ at the current density of $1 \mathrm{~A} / \mathrm{g}$ ), which are higher than those of AC@CB@NiF/G electrode with 10 wt \% CB (AC@CB@NiF/G-10) (96 F/g and $13.3 \mathrm{Wh} / \mathrm{kg}$ ) and AC@G@NiF electrode with $5 \mathrm{wt} \%$ graphene (AC@G@NiF-5) (78.3 F/g and $10.9 \mathrm{Wh} / \mathrm{kg}$ ). However, the property reduce at higher additive amount can be attributed to the planar structure of graphene sheets, which hinder the electrolyte ion transport in electrode material [38]. A symmetric supercapacitor (SSA) fabricated with AC@G@NiF/G-5 electrodes shows excellent cycling stability without any decrease of specific capacitance after 10,000 cycles. This work can be recognized with reference value for researchers for fundamental research and industrial application of graphene-modified supercapacitors or batteries.

\section{Materials and Methods}

\subsection{Materials}

The activated carbon (AC) powder (Kuraray YF-50) was received from Kuraray international trade CO., LTD. (Shanghai, China). The graphene powder (GRF-H-FLGA-01, lateral size 5-10 $\mu \mathrm{m}$, thickness $\sim 2 \mathrm{~nm}$ ) was brought from SZgraphene nanotechnology Company (Suzhou, China). The Potassium hydroxide ( $\mathrm{KOH}, \mathrm{GR}, \geq 85 \mathrm{wt} \%$ ) and N-Methyl pyrrolidone (NMP, AR, $\geq 99.0 \mathrm{wt} \%$ ) were brought from Sinopharm Chemical Reagent Company (Shanghai, China). The nickel foam (NiF, HGP, 110 ppi, $500 \mathrm{~g} / \mathrm{m}^{2}$ ) was obtained from HGP technology CO., LTD (Wuzhou, China). The aqueous membrane (NKK-MPF 30AC-100), Polyvinylidene Fluoride (PVDF, Solvay TA-6020/1001) and carbon black (CB, Ketjenblack EC300J, Lion CO., Tokyo, Japan) were purchased on Taobao (https:/ /item.taobao.com/ item.htm?spm=a230r.1.14.16.73d46067nVvMYK\&id=530857358840\&ns=1\&abbucket=3\#detail).

\subsection{CVD Growth of G/NiF Current Collector}

NiFs were placed in the middle of a quartz tube furnace, and then the furnace should be vacuumized and filled with Ar twice to eliminate the residual air in the furnace. Before growth $\mathrm{NiFs}$ were heated to $1000^{\circ} \mathrm{C}$ in $30 \mathrm{~min}$ under the gas flow of $\mathrm{Ar}(200 \mathrm{sccm})$ and $\mathrm{H}_{2}(200 \mathrm{sccm})$, and then annealed at $1000{ }^{\circ} \mathrm{C}$ for 10 min under the gas flow of $\operatorname{Ar}(200 \mathrm{sccm})$ and $\mathrm{H}_{2}(300 \mathrm{sccm})$, in order to remove the oxide layers on NiFs. Methane $\left(\mathrm{CH}_{4}\right)$ was then injected into the quartz as carbon source to growth graphene under ambient pressure with gas flow of $\mathrm{Ar}(400 \mathrm{sccm}), \mathrm{H}_{2}(200 \mathrm{sccm})$ and $\mathrm{CH}_{4}$ $(100 \mathrm{sccm})$ at $1000{ }^{\circ} \mathrm{C}$. After $10 \mathrm{~min}$, the quartz was fast cooled to room temperature under the gas flow of $\mathrm{Ar}(200 \mathrm{sccm})$ and $\mathrm{H}_{2}(300 \mathrm{sccm})$. After growth of graphene, the color of NiF/Gs changed from shallow to deep.

\subsection{Preparation of AC Electrodes and Assembly of Supercapacitors}

Certain amount of AC, graphene, CB and PVDF were mixed in NMP by a high-shear dispersion homogenizer (U400/80-220, BW Motor Co., Shanghai, China) at $1800 \mathrm{rpm} / \mathrm{min}$ for $4 \mathrm{~h}$ to obtain uniformly dispersed slurries. And then, these slurries were dip coated on $\mathrm{NiF} / \mathrm{G}$ or $\mathrm{NiF}$ current collectors and dried at $130{ }^{\circ} \mathrm{C}$ in vacuum for $24 \mathrm{~h}$ to prepare AC electrodes. The current collectors were made into two specifications (square with $1 \mathrm{~cm} \times 1 \mathrm{~cm}$ and circular piece with diameter $13 \mathrm{~mm}$ ). The square electrodes were used to take electrochemical measurements with a standard three-electrode method. The circular electrodes were used to fabricate symmetrical supercapacitors. Two electrodes with similar mass of electrode materials were assembled into a button capacitor with the membrane (NKK-MPF 30AC-100, Kawasaki, Japan), electrolyte (6 M KOH) and button cell module (CR 2025). 


\subsection{Characterizations and Electrochemical Measurements}

The morphology of NiF/G current collector, raw material powders and AC electrodes was observed using scanning electron microscope (SEM, Quanta FEG 250, FEI Co., Hillsboro, OR, USA). The Raman spectra were carried out by a Raman spectrometer (LabRam HR800-UV-NIR, HORIBA Jobin Yvon, Paris, France, $\lambda=532.15$ nm). The X-ray photoelectron spectroscopy (XPS) spectra were measured by an X-ray photoelectron spectroscopy (Thermo Scientific ESCALAB 250 XI, Waltham, MA, USA). The transmission electron microscopy (TEM, Tecnai G2 F20 S-Twin, FEI Co.) was used to characterize the morphology and layer number of high quality few-layer graphene. The specific surface areas were tested by a specific surface area analyzer (BET) (Micromeritics TriStar II 3020, Micromeritics instrument (Shanghai) LTD., Shanghai, China). The surface resistance of electrode materials was measured by a surface resistance meter (RC2175, EDTM Co., New York, NY, USA). The test of resistivity and resistance of current collectors were carried on by a multifunction digital four-probe tester (ST 2258C, Suzhou Jingge Electronics Co., Suzhou, China). All the electrochemical measurements of electrodes and supercapacitors were performed by an electrochemical workstation (CHI660D, CH Instruments, Inc., Shanghai, China) in $6 \mathrm{M} \mathrm{KOH}$ aqueous solution as the electrolyte, using a standard three-electrode method and two-electrode method, respectively.

\section{Results and Discussion}

AC@G@NiF/G electrodes with different graphene conductive additive amounts of 3-10 wt \% were prepared using $\mathrm{NiF} / \mathrm{Gs}$ as current collectors and few-layer graphene sheets as conductive additive (Figure 1a). The AC@G@NiF/G electrodes show enhanced performances compared with both electrodes on $\mathrm{NiF}$ current collectors and electrodes with common $\mathrm{CB}$ as conductive additive, especially in capacitance and rate capability (Figure 1b,c). Figure $1 \mathrm{~b}$ shows that the specific capacitance of AC@G@NiF/G electrode just with 5 wt \% graphene has exceeded that of AC@CB@NiF/G electrode with $10 \mathrm{wt} \% \mathrm{CB}$, indicating few-layer graphene is potential conductive to realize higher performance at lower additive amount, instead of common CB.

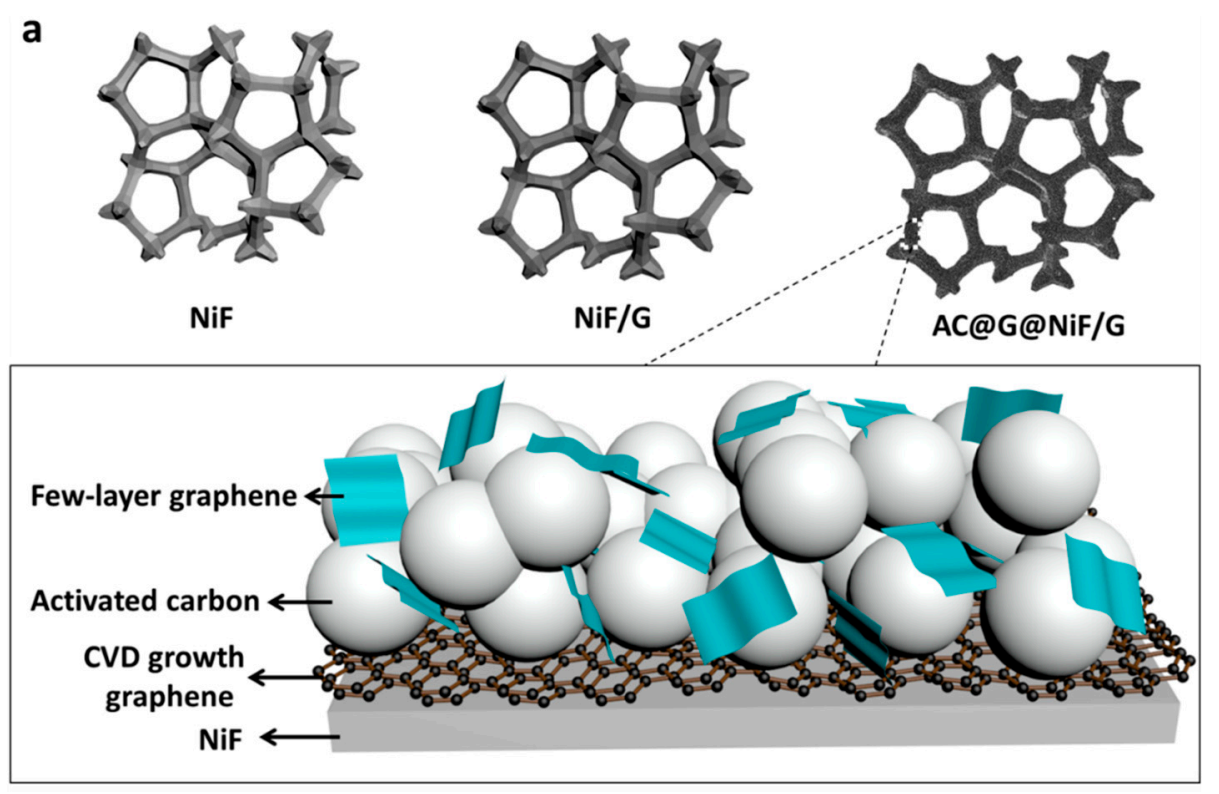

Figure 1. Cont. 

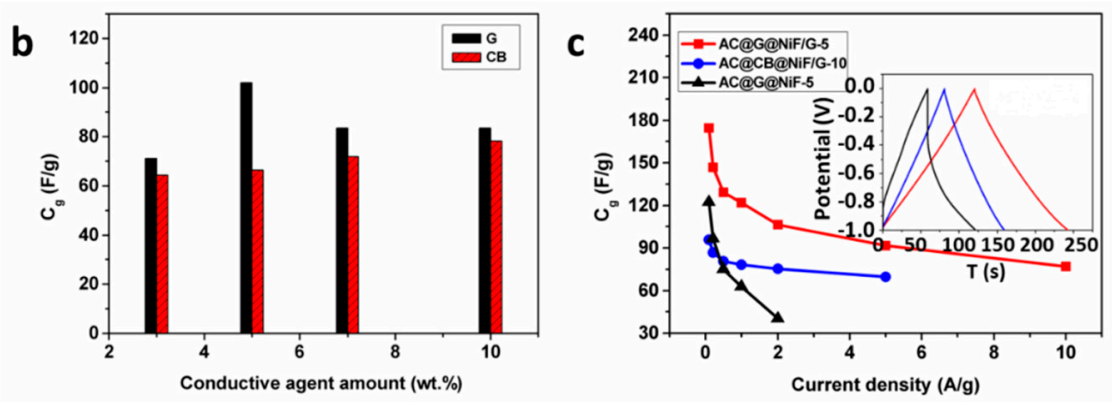

Figure 1. The improved AC@G@NiF/G electrodes. (a) Schematic illustration for the AC@G@NiF/G electrode using graphene-modified $\mathrm{NiF}$ as current collector and few-layer graphene sheets as conductive additive. (b) The specific capacitances of AC@G@NiF/G and AC@CB@NiF/G electrodes with different conductive additive amounts of $3 \mathrm{wt} \%, 5 \mathrm{wt} \%, 7 \mathrm{wt} \%$, and $10 \mathrm{wt} \%$ at the scan rate of $50 \mathrm{mV} / \mathrm{s}$. (c) The specific capacitances of AC@G@NiF/G-5, AC@CB@NiF/G-10, and AC@G@NiF electrodes, as functions of the current densities (the inset shows the corresponding GCD curves at the current density of $1 \mathrm{~A} / \mathrm{g}$, and the black, blue, and red curves represent AC@G@NiF-5, AC@CB@NiF/G-10, and AC@G@NiF/G-5, respectively).

\subsection{The Roles of NiF/G Current Collectors}

The AC@G@NiF/G electrodes show the higher specific capacitances, energy densities and rate capabilities, which can hardly occur without the use of NiF/G current collectors. Graphene can be obviously observed on the surface of $\mathrm{NiF}$ after $\mathrm{CVD}$ growth, which remarkably increases the roughness of the current collector (Figure S1a-c). And electrode materials are well adhered to the $\mathrm{NiF} / \mathrm{G}$ current collectors (Figure S1e-h). Through the Raman spectrum of 3D graphene after liquid etching NiF/G to remove NiF substrate, CVD graphene is supposed with low defects (Figure S1d).

In order to illuminate the contribution of the $\mathrm{NiF} / \mathrm{G}$ current collectors, AC electrodes on common $\mathrm{NiF}$ current collectors with graphene conductive additive of 5 wt \% (AC@G@NiF-5) were made as comparison to AC@G@NiF/G-5 electrodes. Cyclic voltammetry (CV) curves at scan rates of $10-500 \mathrm{mV} / \mathrm{s}$ and galvanostatic charge-discharge (GCD) curves at current densities of $0.1-1 \mathrm{~A} / \mathrm{g}$ within a voltage range of $-1-0 \mathrm{~V}$ of the AC@G@NiF-5 electrode are shown in Figure S2a,b. Combined with the corresponding specific capacitances as functions of scan rates and current densities (Figure $\mathrm{S} 2 \mathrm{c}-\mathrm{f}$ ), it can be confirmed that electrodes on $\mathrm{NiF} / \mathrm{G}$ have higher specific capacitances and much better rate capabilities than those on $\mathrm{NiF}$. At the current density of $1 \mathrm{~A} / \mathrm{g}$, the electrode on $\mathrm{NiF} / \mathrm{G}$ gets the higher specific capacitance and energy density (specific capacitance $123.6 \mathrm{~F} / \mathrm{g}$ and energy density $17.2 \mathrm{Wh} / \mathrm{kg}$ ) than those of electrode on $\mathrm{NiF}$ (specific capacitance $78.3 \mathrm{~F} / \mathrm{g}$ and energy density $10.9 \mathrm{Wh} / \mathrm{kg}$ ). According to the CV curves at the scan rate of $50 \mathrm{mV} / \mathrm{s}$ in Figure S2e, the shape of CV curve of AC@G@NiF/G-5 electrode is more symmetrical and close to an ideal rectangle, which is equivalent to better reversibility of charge-discharge reaction and capacitance characteristic.

This superior rate capability can be attributed to the significantly lowered ESR, which may be derived from the enhanced electrical contact between active materials and current collectors by CVD growth graphene, as evidenced by the electrochemical impedance spectroscopy (EIS) analysis (Figure 2a). The much smaller semicircle in high frequency represents the lower charge-transfer resistance occurring at the electrolyte/electrode interface. The more inclined line in the low frequency of electrode on $\mathrm{NiF} / \mathrm{G}$ indicates the better modified Warburg impedance related to the diffusion impedance. Good cycling stability is an important target in performance enhancement of supercapacitor electrodes. As demonstrated in Figure 2d, the AC@G@NiF/G-5 electrode exhibits a more stable cycle life and has almost no degradation in specific capacitance after 10,000 charge-discharge cycles.

The roles of NiF/G current collectors are considered as three aspects. Firstly, graphene of high specific surface area depositing directly on $\mathrm{NiF}$ as a carbon buffer layer between AC material and $\mathrm{NiF}$, strengthens the adhesion of active material on current collector, and then improves the contact 
at interface. It is consistent with Figure 2c,d, which demonstrates the SEM images of AC electrodes with 5 wt \% graphene additive on $\mathrm{NiF} / \mathrm{G}$ and $\mathrm{NiF}$ current collectors. The active material of the electrode on $\mathrm{NiF} / \mathrm{G}$ has a more sufficient adhesion on the current collector than that on NiF. Secondly, after CVD growth of graphene, highly conductive 3D graphene network on top of NiF frame enhances the conductivity of current collector, which enhances the electrical contact between AC electrode materials and NiF/G current collectors and then facilitates the charge transfer [21]. Thirdly, the CVD growth graphene also protects the $\mathrm{NiF}$ substrates from corrosion of oxygen and water in air. As a result, $\mathrm{NiF}$ /Gs have lower surface oxidation and better chemical stability, which is beneficial to the further cycle life. As proved by the X-ray photoelectron spectroscopy (XPS) spectra of O1s in Figure 3. Natural oxide and hydroxide layer will be formed on the surface of $\mathrm{NiF}$ due to the oxygen and water in the air, corresponding to $\mathrm{NiO}$ at $529.56 \mathrm{eV}, \mathrm{Ni}_{2} \mathrm{O}_{3}$ at $531.93 \mathrm{eV}$, and $\mathrm{Ni}(\mathrm{OH})_{2}$ at $531.07 \mathrm{eV}$ in the $\mathrm{O} 1 \mathrm{~s}$ spectrum of $\mathrm{NiF}$ (Figure 3a). Peaks at $533.48 \mathrm{eV}$ are thought of the adherent $\mathrm{H}_{2} \mathrm{O}$ from air (Figure $3 \mathrm{a}-\mathrm{c}$ ). After the process of CVD growth graphene, the height of $\mathrm{H}_{2} \mathrm{O}$ peaks of $\mathrm{NiF} / \mathrm{Gs}$ obviously decrease and slightly rise up over time of six months (Figure 3b,c), denoting the good water blocking character of graphene cover layer. The weaken $\mathrm{NiO}$ peak and absent $\mathrm{Ni}(\mathrm{OH})_{2}$ peak of the fresh $\mathrm{NiF} / \mathrm{G}$ in Figure $3 \mathrm{~b}$ mean that oxidation and hydroxylation of nickel are suppressed obviously with the introduction of CVD graphene. And over six months, $\mathrm{NiO}$ and $\mathrm{Ni}(\mathrm{OH})_{2}$ only appear slightly. The reduction of oxide and hydroxide layer will further improve the electrical contact between active materials and current collectors. It is worth noting that the CVD process not only grows the graphene cover layer but also removes the oxide and hydroxide layer on the top of $\mathrm{NiF}$, which reduces resistivity (from $0.38 \mathrm{~m} \Omega \cdot \mathrm{cm}$ of $\mathrm{NiF}$ to $0.358 \mathrm{~m} \Omega \cdot \mathrm{cm}$ of $\mathrm{NiF} / \mathrm{G})$.
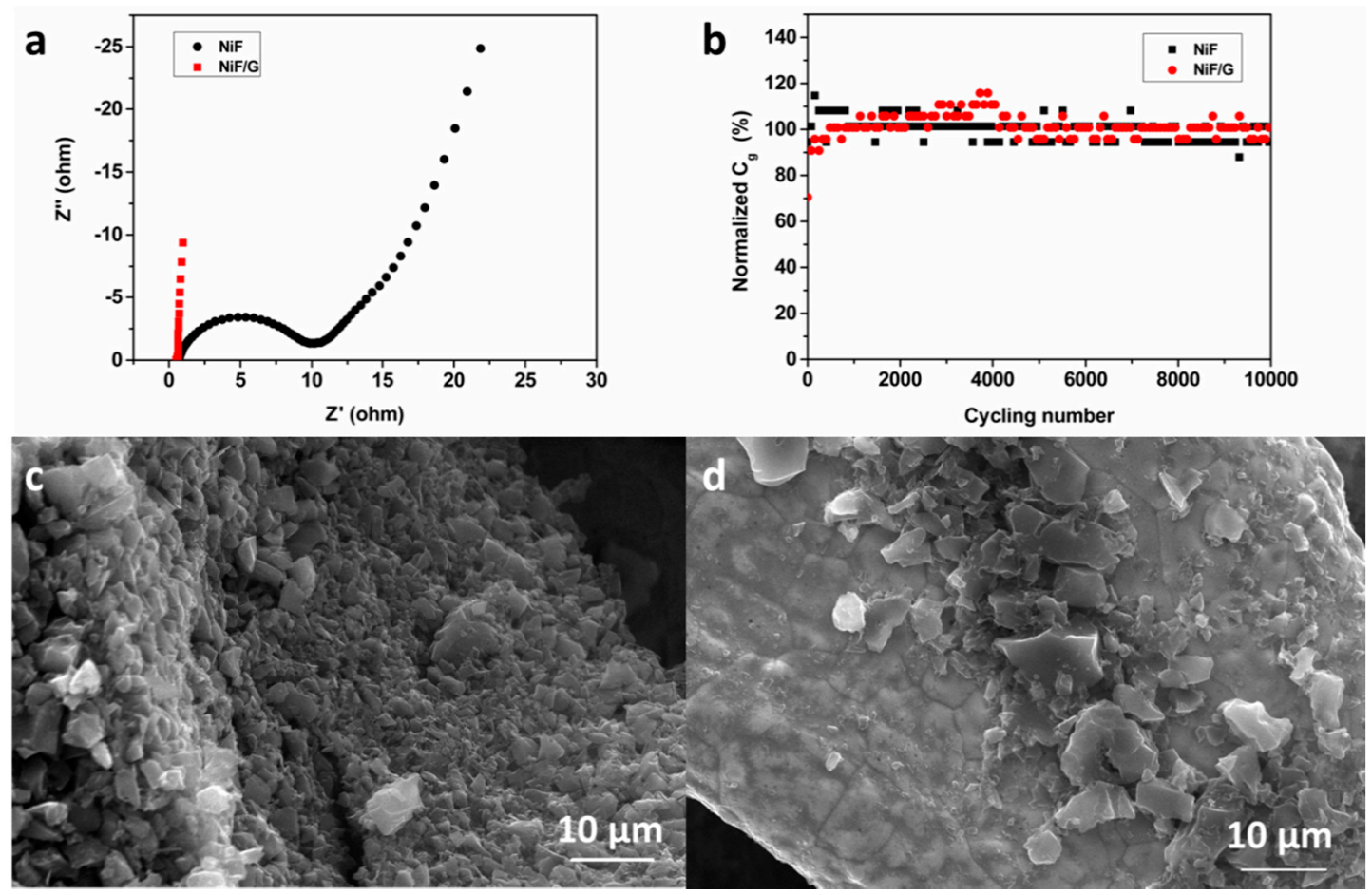

Figure 2. The AC@G@NiF/G-5 electrode and AC@G@NiF-5 electrode. (a) The Nyquist plots. (b) The capacitance retention of 10,000 cycles. (c,d) SEM images of AC@G@NiF/G-5 electrode and AC@G@NiF-5 electrode, respectively (scale bar = $10 \mu \mathrm{m}$ ).

All these three points about the graphene buffer layer have a high conductivity 3D network and chemical stability which guarantees the superior electrochemical performance of the AC@G@NiF/G-5 electrode, such as higher specific capacitance, energy density, better rate capability, cycling stability, and lower resistance. 

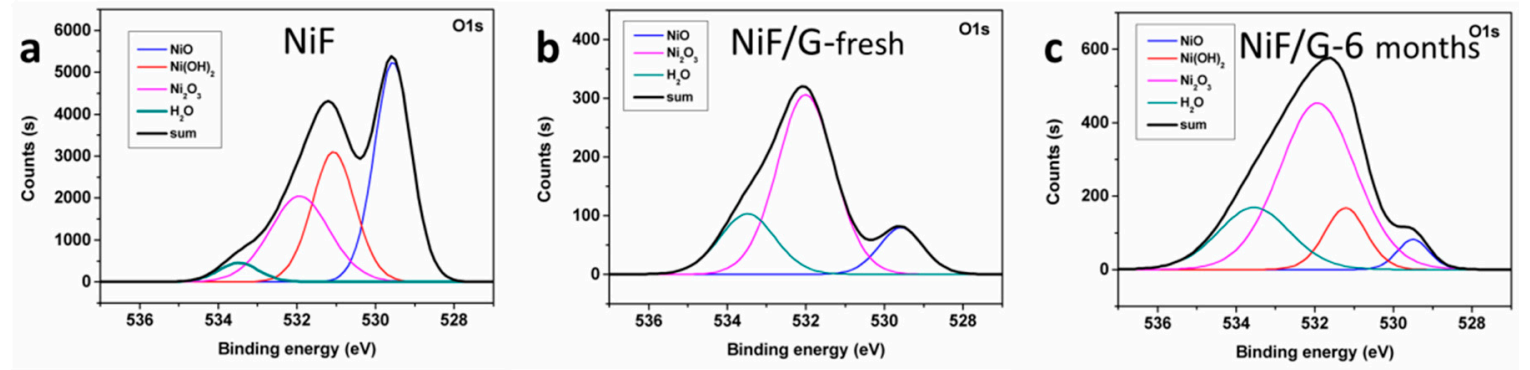

Figure 3. The X-ray photoelectron spectroscopy (XPS) spectra of $\mathrm{NiF}$ and $\mathrm{NiF} / \mathrm{G}$. The fitting O1s XPS spectra of (a) NiF, (b) NiF/G-fresh (just prepared several hours before), and (c) NiF/G-6 months (placing in air for six months).

\subsection{The Effect of Graphene Conductive Additive}

The electrochemical properties of AC@G@NiF/G electrodes with graphene conductive additive varying from $3 \mathrm{wt} \%$ to $10 \mathrm{wt} \%$ are demonstrated in Figure 4 . CV curves at the scan rate of $50 \mathrm{mV} / \mathrm{s}$ and GCD curves at the current density of $1 \mathrm{~A} / \mathrm{g}$ within a voltage range from -1 to $0 \mathrm{~V}$ were measured to evaluate the mass specific capacitance of AC@G@NiF/G electrodes (Figure 2a,b). It was found that with the increase of graphene conductive additive, the electrochemical properties get improved first, which can be attributed to highly conductive graphene improving electrical microenvironment in AC active material of the composite electrodes, facilitating electrolyte ion diffusion and charge transfer in AC, and then promoting the value of specific capacitances. While beyond the graphene additive amount of $5 \mathrm{wt} \%$, specific capacitance values show slight decrease. Because the planar structure of graphene sheets will tend to block the transport of electrolyte ions in electrode materials and impede the transfer of charge. It can be proved by the Nyquist plots in Figure $4 \mathrm{~d}$. The high-frequency intercept on the real axis in the Nyquist plots represents ohmic resistance, including the ionic resistance of electrolyte, the contact resistance between active material and current collector, and the intrinsic resistance of active material. The result clearly reflects that the addition of graphene effectively reduces contact resistances between $\mathrm{AC}$ materials and $\mathrm{NiF} / \mathrm{G}$ current collectors and the intrinsic resistance of AC electrode materials for these AC@G@NiF/G electrodes. Moreover, the slopes in low frequency region also reflect the Warburg impedance, related to the electrolyte ion diffusion in electrodes, indicating that the ion diffusion is enhanced first and then reduced with the increase of graphene. The highest specific capacitance was observed in the AC@G@NiF/G-5 electrode, which was confirmed with both the specific capacitance $102.0 \mathrm{~F} / \mathrm{g}$ at the scan rate of $50 \mathrm{mV} / \mathrm{s}$ and $123.6 \mathrm{~F} / \mathrm{g}$ at the current density of $1 \mathrm{~A} / \mathrm{g}$. The similar law is also obtained in the rate capabilities (Figure 4c), corresponding to the mass specific capacitances of AC@G@NiF/G electrodes as functions of current densities. The similar trend is also found in the BET specific surface areas of AC electrode materials. With the graphene additive amount increasing from $3 \mathrm{wt} \%$ to $10 \mathrm{wt} \%$, the BET specific surface area increases first, and then decreases (Figures S3 and S4). But the peak of BET specific surface area is at $7 \mathrm{wt} \%$ rather than $5 \mathrm{wt} \%$ as the peak of electrochemical properties. It can be supposed that the planar structure of graphene sheets will block the electrolyte ion transport in electrode materials [38]. Although graphene additive helps enhance the BET specific surface area, when graphene conductive additive amount is beyond $5 \mathrm{wt} \%$, we inferred the block effect does more than specific surface area. 

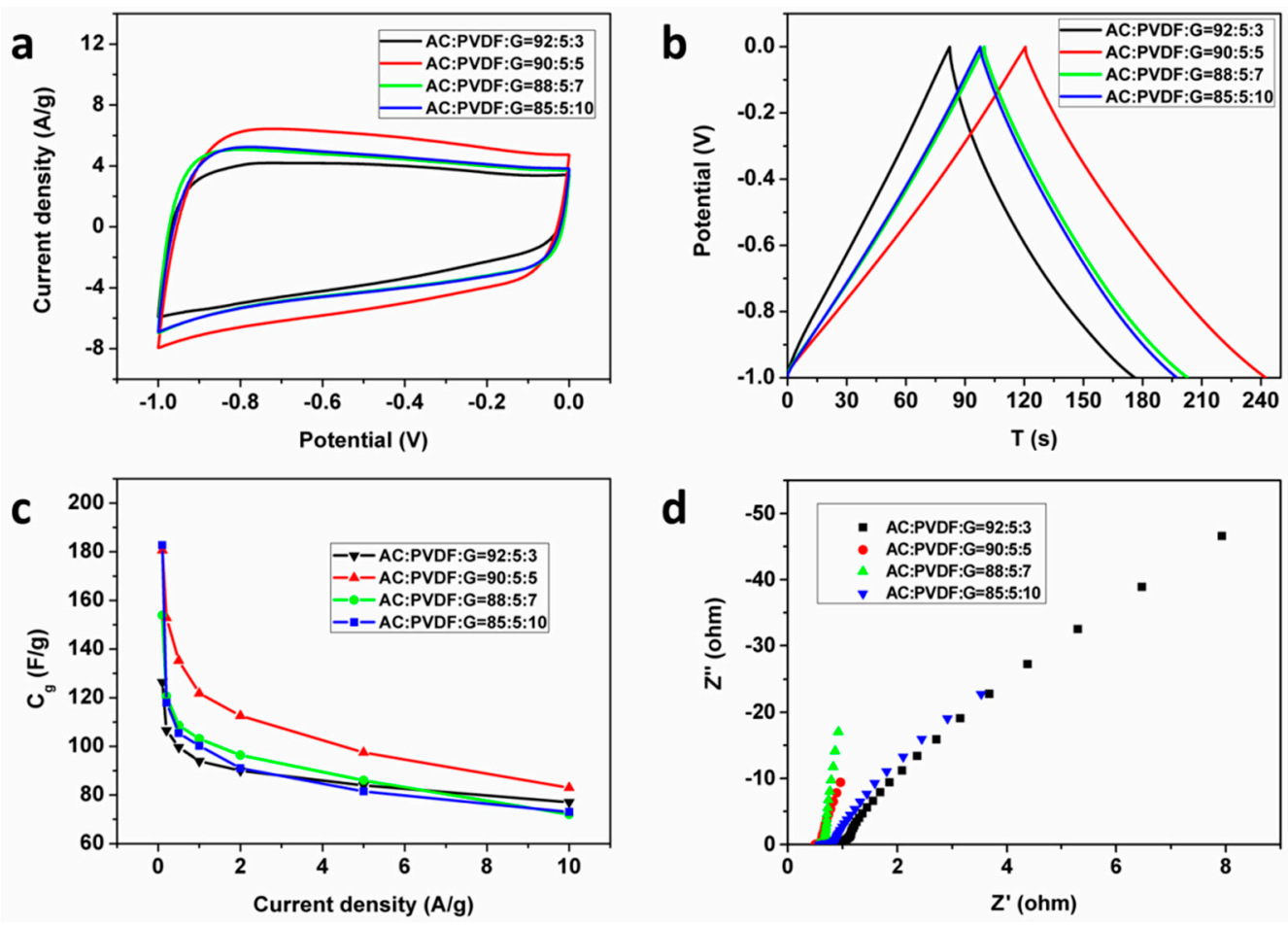

Figure 4. The electrical properties of AC@G@NiF/G electrodes with different graphene conductive additive amounts from 3 to $10 \mathrm{wt} \%$. (a) CV curves at the scan rate of $50 \mathrm{mV} / \mathrm{s}$. (b) GCD curves at the current density of $1 \mathrm{~A} / \mathrm{g}$. (c) The corresponding mass specific capacitances as functions of current densities. (d) The Nyquist plots. The legends in figures represent the different AC@G@NiF/G electrodes with different contents of electrode material components (active material AC, binder PVDF, and conductive additive graphene).

Few-layer graphene nanosheets were used as conductive additive instead of traditional CB to prepare the AC@G@NiF/G composite electrodes, which plays an important role in performance enhancement of electrodes. As a contrast, $\mathrm{AC}$ electrodes on $\mathrm{NiF} / \mathrm{G}$ current collectors with $\mathrm{CB}$ as conductive additive (AC@CB@NiF/G electrodes) were made by the same method as AC@G@NiF/G electrodes. Figures S5-S7 show the Raman spectrum, morphology and surface resistance of AC@G@NiF/G and AC@CB@NiF/G electrodes, which reveals that electrode with graphene as conductive agent has higher conductivity than that of $\mathrm{CB}$ under the same additive amount. It can be attributed to the high quality of few-layer graphene (5-10 layers), which is also proved by the characterizations of the graphene raw material (Figure S8).

The $\mathrm{CV}$ curves at the scan rate of $50 \mathrm{mV} / \mathrm{s}$ and corresponding specific capacitances as functions on scan rates of $\mathrm{AC} @ \mathrm{CB} @ N i F / G$ electrodes with different $\mathrm{CB}$ additive amount of 3-10 wt \% are demonstrated in Figure 5a,b, which indicates that specific capacitances of AC@CB@NiF/G electrodes are improved with the increase of CB conductive additive. The AC@CB@NiF/G-10 electrode has the highest specific capacitance and best rate capability among electrodes with $\mathrm{CB}$. However, it is yet inferior to the AC@G@NiF/G-5 electrode, both in specific capacitances and rate capabilities (Figure S9 and Figure $5 c, d)$. It can be seen from the CV and GCD curves of AC@CB@NiF/G-10 electrode (Figure S10) and those of AC@G@NiF/G-5 electrode (Figure S9a,c). This is because the usage of graphene conductive additive makes the electrode with better conductivity, improves the electrical contact at the electrolyte/electrode interface and facilitates the electrolyte ion diffusion and charge transport.

Table 1 lists some previous works and our graphene/AC composite electrodes for supercapacitors. Our electrode displays much better cycling stability than other graphene/AC composite electrodes and possesses the capacitance commensurate to current level. 
a
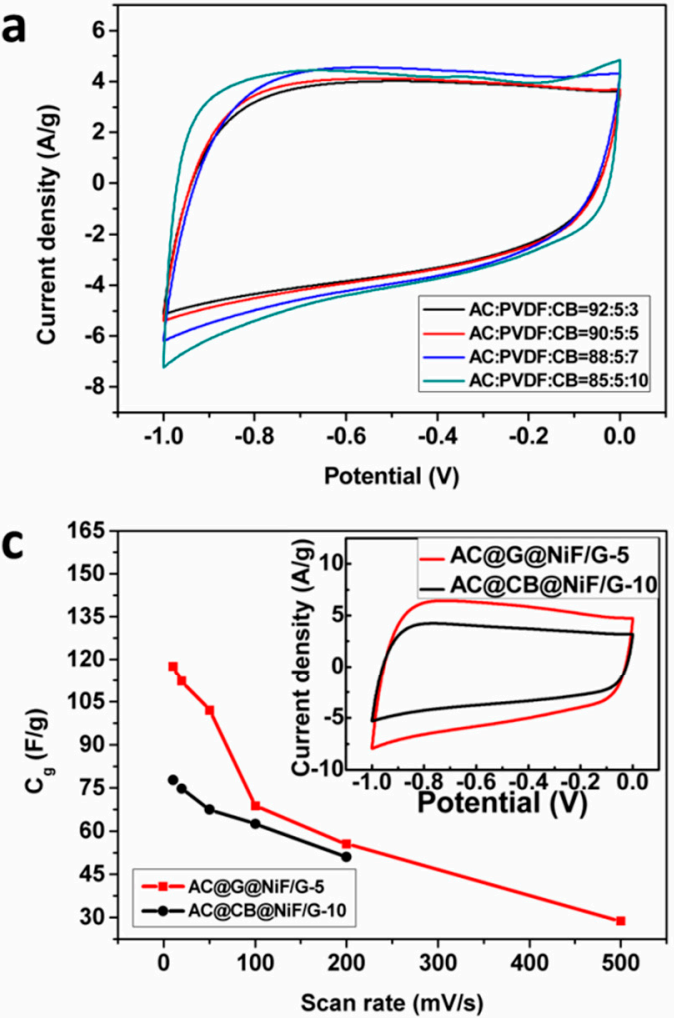

b

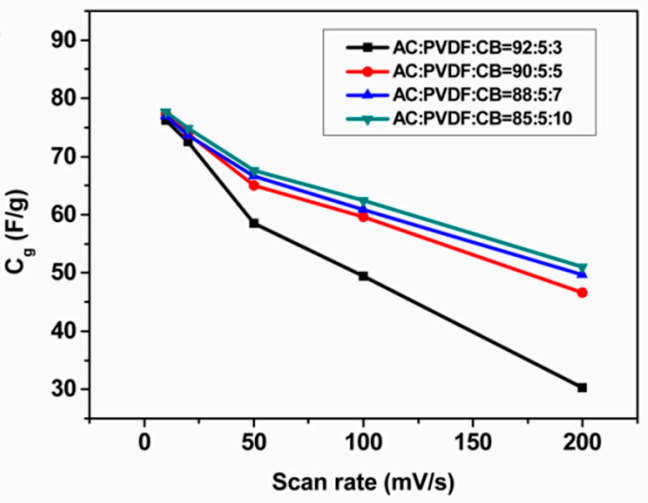

d

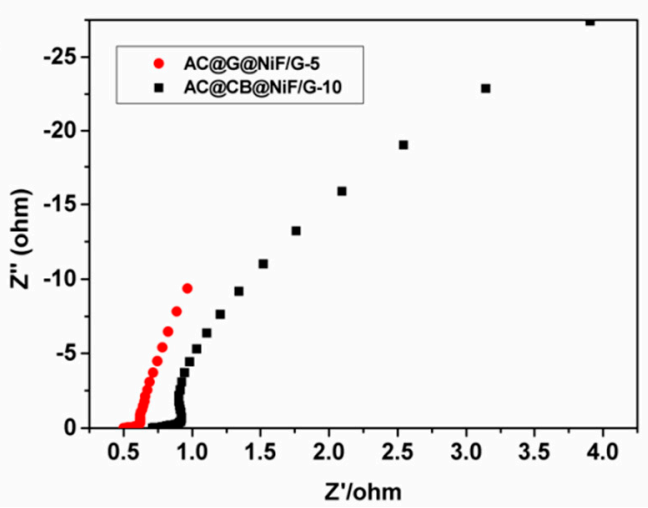

Figure 5. Comparisons of AC@G@NiF/G and AC@CB@NiF/G electrodes. (a) CV curves at the scan rate of $50 \mathrm{mV} / \mathrm{s}$, and (b) the corresponding mass specific capacitances as functions of scan rates of AC@CB@NiF/G electrodes with different $\mathrm{CB}$ amounts. The legends in $(\mathbf{a})$ and $(\mathbf{b})$ represent the different AC@G@NiF/G electrodes with different contents of electrode material components (active material AC, binder PVDF, and conductive additive graphene). (c) The mass specific capacitances as functions of scan rates (The inset shows the $\mathrm{CV}$ curves at the scan rate of $50 \mathrm{mV} / \mathrm{s}$ ), and (d) the Nyquist plots of AC@G@NiF/G-5 and AC@CB@NiF/G-10 electrodes.

Table 1. Electrochemical performances of graphene/AC composite electrodes [39].

\begin{tabular}{|c|c|c|c|c|c|c|}
\hline Electrode & Role of Graphene & Electrolyte & $\begin{array}{l}\text { Specific } \\
\text { Capacitance }\end{array}$ & $\begin{array}{c}\text { Capacity } \\
\text { Retention } \\
\text { Ratio }\end{array}$ & $\begin{array}{l}\text { Energy } \\
\text { Density } \\
\text { (Wh/kg) }\end{array}$ & $\begin{array}{l}\text { Power } \\
\text { Density } \\
\text { (W/kg) }\end{array}$ \\
\hline Graphene/AC [36] & $\begin{array}{l}\text { component of } \\
\text { active material }\end{array}$ & $6 \mathrm{M} \mathrm{KOH}$ & $\begin{array}{l}210 \mathrm{~F} / \mathrm{g} \\
(1 \mathrm{mV} / \mathrm{s})\end{array}$ & $94.7 \%(5000)$ & 22.3 & 33.2 \\
\hline Graphene/AC [40] & $\begin{array}{l}\text { component of } \\
\text { active material }\end{array}$ & $\mathrm{KOH}$ & $122 \mathrm{~F} / \mathrm{g}$ & $90 \%(3000)$ & 6.1 & - \\
\hline $\begin{array}{c}\text { Graphene/AC fiber } \\
\text { [41] }\end{array}$ & dispersant \& binder & $1 \mathrm{M} \mathrm{H}_{2} \mathrm{SO}_{4}$ & $43.8 \mathrm{~F} / \mathrm{g}$ & $\begin{array}{c}90.4 \% \\
(10,000)\end{array}$ & - & - \\
\hline Graphene/AC [42] & $\begin{array}{l}\text { component of } \\
\text { active material }\end{array}$ & $6 \mathrm{M} \mathrm{KOH}$ & $\begin{array}{c}297 \mathrm{~F} / \mathrm{g} \\
(0.1 \mathrm{~A} / \mathrm{g}) \\
\end{array}$ & $90 \%(6000)$ & 6.12 & 4660 \\
\hline $\begin{array}{l}\text { Nitrogen-doped } \\
\text { graphene/AC [43] }\end{array}$ & $\begin{array}{l}\text { component of active } \\
\text { material }\end{array}$ & $6 \mathrm{M} \mathrm{KOH}$ & $\begin{array}{c}145 \mathrm{~F} / \mathrm{g} \\
(20 \mathrm{mV} / \mathrm{s})\end{array}$ & $98.4 \%(5000)$ & - & - \\
\hline $\begin{array}{c}\text { AC@G@NiF/G [our } \\
\text { work] }\end{array}$ & $\begin{array}{l}\text { Conductive agent \& } \\
\text { modification of } \\
\text { current collectors }\end{array}$ & $6 \mathrm{M} \mathrm{KOH}$ & $\begin{array}{c}123.6 \mathrm{~F} / \mathrm{g} \\
(1 \mathrm{~A} / \mathrm{g})\end{array}$ & $\begin{array}{c}>95 \% \\
(10,000)\end{array}$ & 17.2 & 507.5 \\
\hline
\end{tabular}

\subsection{The Symmetric Supercapacitor Assembled by AC@G@NiF/G-5 Electrodes}

Two AC@G@NiF/G-5 electrodes with the same mass of the active material were assembled to a symmetric supercapacitor (SSC) with the electrolyte being a $6 \mathrm{M} \mathrm{KOH}$ aqueous solution. The CV curves 
Raman 10-500 mV/s shown in Figure 6a exhibit quasi-rectangular shapes, even at a high scan rate of $500 \mathrm{mV} / \mathrm{s}$, suggesting good reversibility and typical electrical double-layer capacitive characteristics of our device. The GCD curves at various current densities of $0.1-10 \mathrm{~A} / \mathrm{g}$ in the potential range of $0-1 \mathrm{~V}$ are plotted in Figure 6c. The quasi-equicrural triangle curves also indicate a good capacitive behavior for the supercapacitor. Excellent cycling stability is demonstrated by Figure $6 \mathrm{f}$ displaying almost no decrease of capacitance after 10,000 cycles. Furthermore, the Nyquist plots of the SSC before and after cycling in Figure 6e demonstrate that a little bigger curvature of semicircle and left shift of intersection of the horizontal axis in the high frequency region, which reveal the almost unchanged ESR after cycling. The slop larger than 1 in the low frequency region reflects the well held capacitive behavior after cycling, which can be attributed to the further sufficient immersion of electrolyte after cycling.
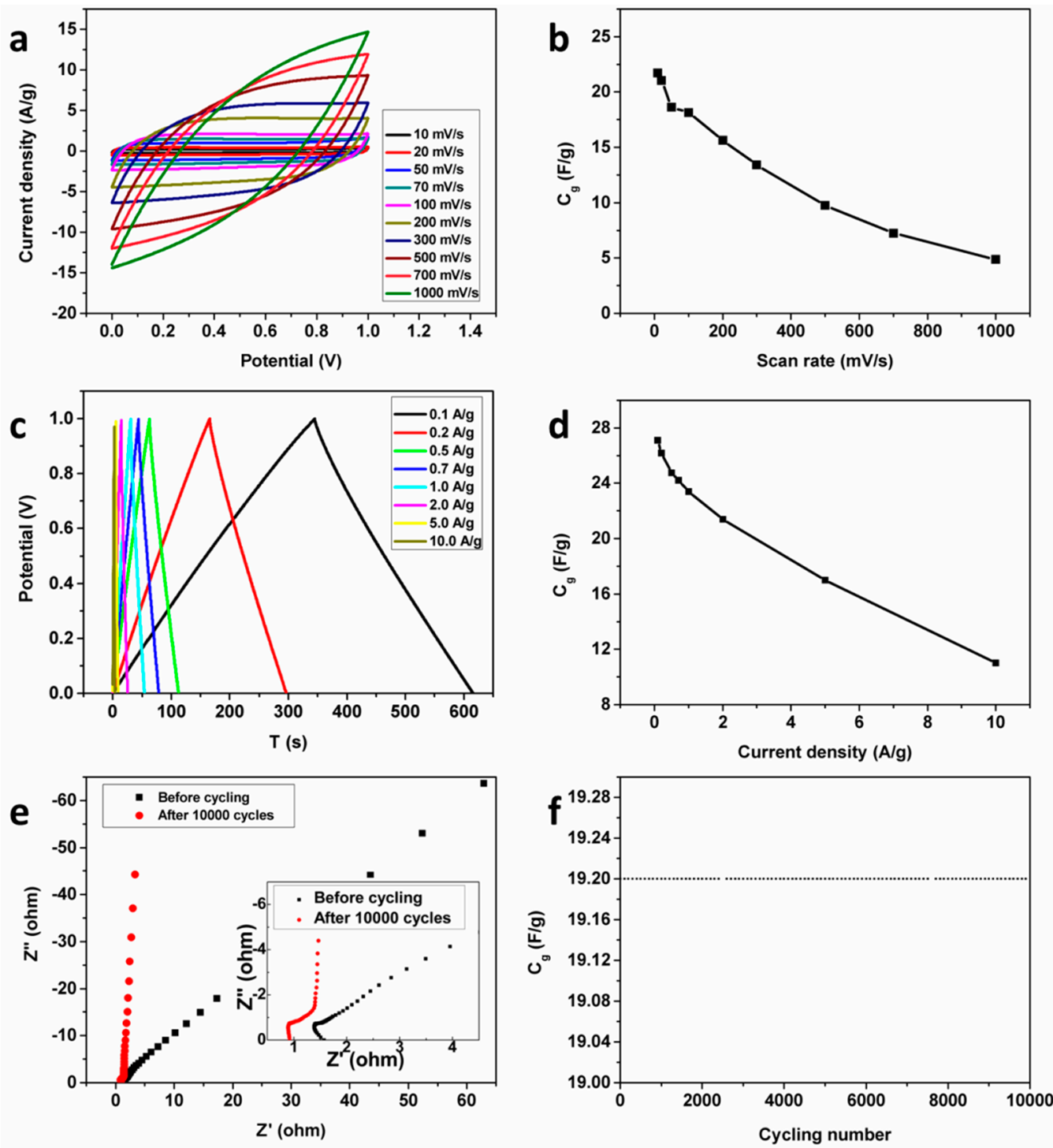

Figure 6. The electrochemical properties of the symmetric supercapacitor assembled by AC@G@NiF/G-5 electrodes. (a) CV curves at scan rates of 10-1000 mV/s. (b) The corresponding mass specific capacitances as a function of scan rates. (c) GCD curves at different current densities of $0.1-10 \mathrm{~A} / \mathrm{g}$. (d) The corresponding mass specific capacitances as a function of current densities of the SSC assembled by AC@G@NiF/G-5 electrodes. (e) The Nyquist plots before and after cycling for 10,000 times (The inset is the enlarged image of high frequency region). (f) The capacitance retention of 10,000 cycles. 


\section{Conclusions}

In summary, we demonstrated a kind of performance-enhanced electrodes of supercapacitors involved in NiF/G current collectors and graphene as the conductive additive. Firstly, the NiF/G current collectors guarantee the conductivity and stability of the current collectors and strengthen the adhesion with active materials, which provide a much more effective electrical contact for fast charge transfer from active materials to current collectors. Secondly, the addition of graphene as the conductive additive directly improves the conductivity of the electrode. Both the NiF/G current collectors and the highly conductive graphene additive make the performances of as-prepared AC@G@NiF/G electrodes superior to those of AC@CB@NiF/G and AC@G@NiF electrodes. Just at low addition of graphene conductive additive of $5 \mathrm{wt} \%$, the AC@G@NiF/G-5 electrodes show best electrochemical properties, specific capacitance $123.6 \mathrm{~F} / \mathrm{g}$ and energy density $17.2 \mathrm{Wh} / \mathrm{kg}$ at the current density of 1 A/g. The SSA assembled by AC@G@NiF/G-5 electrodes also shows excellent cycling stability with almost no decrease of specific capacitance after 10,000 cycles. The results are expected with reference value for researchers for fundamental research and industrial application of graphene-modified supercapacitors or even batteries.

Supplementary Materials: The following are available online at http:/ /www.mdpi.com/1996-1944/11/5/799/ s1.

Author Contributions: R.W., W.L. and L.L. conceived of the study. R.W. carried out the experiments and wrote the manuscript. Y.Q. measured and analyzed the XPS spectra. W.L., S.Z., F.L., Y.G., M.C., Q.L. and L.L. revised the manuscript. All authors discussed the results and commented on the manuscript before submission.

Funding: This work was supported by the National Natural Science Foundation of China [Grant Nos. 11474310 and 61605237], the State Key Program of National Natural Science Foundation of China [Grant No. 61734008], and the projects of Jiangsu province and Suzhou city [Grant Nos. BE2016006-3, BK20150366, BK20150367, 1501131B, and SYG201629].

Acknowledgments: The authors are also grateful for the technical support of Nano-X, the Platforms of Characterization \& Test, and Nanofabrication Facility from Suzhou Institute of Nano-Tech and Nano-Bionics.

Conflicts of Interest: The authors declare no conflict of interests.

\section{References}

1. Sengottaiyan, C.; Jayavel, R.; Bairi, P.; Shrestha, R.G.; Ariga, K.; Shrestha, L.K. Cobalt Oxide/Reduced Graphene Oxide Composite with Enhanced Electrochemical Supercapacitance Performance. Bull. Chem. Soc. Jpn. 2017, 90, 955-962. [CrossRef]

2. Jiang, L.; Yan, J.; Zhou, Y.; Hao, L.; Xue, R.; Jiang, L.; Yi, B. Activated Carbon/Graphene Composites with High-Rate Performance as Electrode Materials for Electrochemical Capacitors. J. Solid State Electrochem. 2013, 17, 2949-2958. [CrossRef]

3. Bose, S.; Kuila, T.; Mishra, A.K.; Rajasekar, R.; Kim, N.H.; Lee, J.H. Carbon-Based Nanostructured Materials and Their Composites as Supercapacitor Electrodes. J. Mater. Chem. 2012, 22, 767-784. [CrossRef]

4. Wang, J.G.; Wei, B. Special Issue: Materials for Electrochemical Capacitors and Batteries. Materials 2017, 10, 438. [CrossRef] [PubMed]

5. Cakici, M.; Kakarla, R.R.; Alonso-Marroquin, F. Advanced Electrochemical Energy Storage Supercapacitors Based on the Flexible Carbon Fiber Fabric-Coated with Uniform Coral-Like $\mathrm{MnO}_{2}$ Structured Electrodes. Chem. Eng. J. 2017, 309, 151-158. [CrossRef]

6. Liu, M.; Shi, M.; Lu, W.; Zhu, D.; Li, L.; Gan, L. Core-Shell Reduced Graphene Oxide/MnOx@Carbon Hollow Nanospheres for High Performance Supercapacitor Electrodes. Chem. Eng. J. 2017, 313, 518-526. [CrossRef]

7. Wang, Y.; Mayorga-Martinez, C.C.; Pumera, M. Polyaniline/MoSX Supercapacitor by Electrodeposition. Bull. Chem. Soc. Jpn. 2017, 90, 847-853. [CrossRef]

8. Yu, M.H.; Lin, D.; Feng, H.B.; Zeng, Y.X.; Tong, Y.X.; Lu, X.H. Boosting the Energy Density of Carbon-Based Aqueous Supercapacitors by Optimizing the Surface Charge. Angew. Chem. Int. Ed. 2017, 56, 5454-5459. [CrossRef] [PubMed] 
9. Zhu, Y.W.; Murali, S.; Stoller, M.D.; Ganesh, K.J.; Cai, W.W.; Ferreira, P.J.; Pirkle, A.; Wallace, R.M.; Cychosz, K.A.; Thommes, M.; et al. Carbon-Based Supercapacitors Produced by Activation of Graphene. Science 2011, 332, 1537-1541. [CrossRef] [PubMed]

10. Lin, T.Q.; Chen, I.W.; Liu, F.X.; Yang, C.Y.; Bi, H.; Xu, F.F.; Huang, F.Q. Nitrogen-Doped Mesoporous Carbon of Extraordinary Capacitance for Electrochemical Energy Storage. Science 2015, 350, 1508-1513. [CrossRef] [PubMed]

11. Pan, Z.H.; Liu, M.N.; Yang, J.; Qiu, Y.C.; Li, W.F.; Xu, Y.; Zhang, X.Y.; Zhang, Y.G. High Electroactive Material Loading on a Carbon Nanotube@3D Graphene Aerogel for High-Performance Flexible All-Solid-State Asymmetric Supercapacitors. Adv. Funct. Mater. 2017, 27. [CrossRef]

12. Khan, A.H.; Ghosh, S.; Pradhan, B.; Dalui, A.; Shrestha, L.K.; Acharya, S.; Ariga, K. Two-Dimensional (2D) Nanomaterials Towards Electrochemical Nanoarchitectonics in Energy-Related Applications. Bull. Chem. Soc. Jpn. 2017, 90, 627-648. [CrossRef]

13. Suen, N.T.; Hung, S.F.; Quan, Q.; Zhang, N.; Xu, Y.J.; Chen, H.M. Electrocatalysis for the Oxygen Evolution Reaction: Recent Development and Future Perspectives. Chem. Soc. Rev. 2017, 46, 337-365. [CrossRef] [PubMed]

14. Xu, J.; Wang, Y.; Hu, S. Nanocomposites of Graphene and Graphene Oxides: Synthesis, Molecular Functionalization and Application in Electrochemical Sensors and Biosensors. A Review. Microchim. Acta 2017, 184, 1-44. [CrossRef]

15. Xu, Y.; Li, J.; Huang, W. Porous Graphene Oxide Prepared on Nickel Foam by Electrophoretic Deposition and Thermal Reduction as High-Performance Supercapacitor Electrodes. Materials 2017, 10, 936. [CrossRef]

16. Ning, J.; Zhang, T.; He, Y.; Jia, C.; Saha, P.; Cheng, Q. $\mathrm{Co}_{3} \mathrm{O}_{4} @$ CoS Core-Shell Nanosheets on Carbon Cloth for High Performance Supercapacitor Electrodes. Materials 2017, 10, 608. [CrossRef] [PubMed]

17. Zhao, X.; Li, W.; Chen, H.; Wang, S.; Kong, F.; Liu, S. Facile Control of the Porous Structure of Larch-Derived Mesoporous Carbons via Self-Assembly for Supercapacitors. Materials 2017, 10, 1330. [CrossRef] [PubMed]

18. Gao, W.; Singh, N.; Song, L.; Liu, Z.; Reddy, A.L.M.; Ci, L.J.; Vajtai, R.; Zhang, Q.; Wei, B.Q.; Ajayan, P.M. Direct Laser Writing of Micro-Supercapacitors on Hydrated Graphite Oxide Films. Nat. Nanotechnol. 2011, 6, 496-500. [CrossRef] [PubMed]

19. Pachfule, P.; Shinde, D.; Majumder, M.; Xu, Q. Fabrication of Carbon Nanorods and Graphene Nanoribbons from a Metal-Organic Framework. Nat. Chem. 2016, 8, 718-724. [CrossRef] [PubMed]

20. Xie, Y.B.; Zhan, Y.Y. Electrochemical Capacitance of Porous Reduced Graphene Oxide/Nickel Foam. J. Porous Mat. 2015, 22, 403-412. [CrossRef]

21. Cao, X.H.; Shi, Y.M.; Shi, W.H.; Lu, G.; Huang, X.; Yan, Q.Y.; Zhang, Q.C.; Zhang, H. Preparation of Novel 3D Graphene Networks for Supercapacitor Applications. Small 2011, 7, 3163-3168. [CrossRef] [PubMed]

22. Ortúzar, M.; Moreno, J.; Dixon, J. Ultracapacitor-Based Auxiliary Energy System for an Electric Vehicle: Implementation and Evaluation. IEEE. Trans. Ind. Electron. 2007, 54, 2147-2156. [CrossRef]

23. Yu, H.; Tang, Q.; Wu, J.; Lin, Y.; Fan, L.; Huang, M.; Lin, J.; Li, Y.; Yu, F. Using Eggshell Membrane as a Separator in Supercapacitor. J. Power Sources 2012, 206, 463-468. [CrossRef]

24. Fan, X.Y.; Ke, F.S.; Wei, G.Z.; Huang, L.; Sun, S.G. Sn-Co Alloy Anode Using Porous Cu as Current Collector for Lithium Ion Battery. J. Alloy Compd. 2009, 476, 70-73. [CrossRef]

25. Arulepp, M.; Permann, L.; Leis, J.; Perkson, A.; Rumma, K.; Jänes, A.; Lust, E. Influence of the Solvent Properties on the Characteristics of a Double Layer Capacitor. J. Power Sources 2004, 133, 320-328. [CrossRef]

26. Yang, C.P.; Yin, Y.X.; Zhang, S.F.; Li, N.W.; Guo, Y.G. Accommodating Lithium into 3D Current Collectors with a Submicron Skeleton towards Long-Life Lithium Metal Anodes. Nat. Commun. 2015, 6, 8058. [CrossRef] [PubMed]

27. Tang, X.X.; Liu, W.; Ye, B.Y.; Tang, Y. Preparation of Current Collector with Blind Holes and Eenhanced Cycle Performance of Silicon-Based Anode. Trans. Nonferr. Met. Soc. 2013, 23, 1723-1727. [CrossRef]

28. Kim, S.W.; Kim, I.H.; Kim, S.I.; Jang, J.H. Nickel Hydroxide Supercapacitor with a Theoretical Capacitance and High Rate Capability Based on Hollow Dendritic 3D-Nickel Current Collectors. Chem. Asian J. 2017, 12, 1291-1296. [CrossRef] [PubMed]

29. Xing, W.; Qiao, S.Z.; Wu, X.Z.; Gao, X.L.; Zhou, J.; Zhuo, S.P.; Hartono, S.B.; Hulicova-Jurcakova, D. Exaggerated Capacitance Using Electrochemically Active Nickel Foam as Current Collector in Electrochemical Measurement. J. Power Sources 2011, 196, 4123-4127. [CrossRef] 
30. Ramabadran, U.; Ryan, G.; Zhou, X.; Farhat, S.; Manciu, F.; Tong, Y.; Ayler, R.; Garner, G. Reduced Graphene Oxide on Nickel Foam for Supercapacitor Electrodes. Materials 2017, 10, 1295. [CrossRef] [PubMed]

31. Gu, W.; Fan, X.M.; Yao, J.L.; Ren, B.; Gu, R.A.; Tian, Z.Q. Investigation on Surface-Enhanced Raman Scattering Activity on an Ex Situ ORC Roughened Nickel Electrode. J. Raman Spectrosc. 2009, 40, 405-410. [CrossRef]

32. Wang, L.Z.; Fang, H.; Gu, S.H.; Zhang, L.S. The Performance of EDLC Using Chemical Etched Nickel Foil Current Collector. Battery Bimon. 2010, 40, 317-319.

33. Gong, K.; Huang, Y.H.; Tian, Z.J.; Liu, Z.D.; Wang, G.F. Preparation of Porous Metal Nickel Used for Current Collector with Jet Electrodeposition and Electrochemical Capacitor Properties. Mater. Mech. Eng. 2011, 35, 62-69.

34. Xie, K.Y.; Wei, W.F.; Yuan, K.; Lu, W.; Guo, M.; Li, Z.H.; Song, Q.; Liu, X.R.; Wang, J.G.; Shen, C. Toward Dendrite-Free Lithium Deposition via Structural and Interfacial Synergistic Effects of 3D Graphene@Ni Scaffold. ACS Appl. Mater. Inter. 2016, 8, 26091-26097. [CrossRef] [PubMed]

35. Toupin, M.; Bélanger, D.; Hill, I.R.; Quinn, D. Performance of Experimental Carbon Blacks in Aqueous Supercapacitors. J. Power Sources 2005, 140, 203-210. [CrossRef]

36. Zheng, C.; Zhou, X.; Cao, H.; Wang, G.; Liu, Z. Synthesis of Porous Graphene/Activated Carbon Composite with High Packing Density and Large Specific Surface Area for Supercapacitor Electrode Material. J. Power Sources 2014, 258, 290-296. [CrossRef]

37. Raccichini, R.; Varzi, A.; Passerini, S.; Scrosati, B. The Role of Graphene for Electrochemical Energy Storage. Nat. Mater. 2015, 14, 271-279. [CrossRef] [PubMed]

38. Su, F.Y.; He, Y.B.; Li, B.; Chen, X.C.; You, C.H.; Wei, W.; Lv, W.; Yang, Q.H.; Kang, F. Could Graphene Construct an Effective Conducting Network in a High-Power Lithium Ion Battery? Nano Energy 2012, 1, 429-439. [CrossRef]

39. Kandasamy, S.K.; Kandasamy, K. Recent Advances in Electrochemical Performances of Graphene Composite (Graphene-Polyaniline/Polypyrrole/Activated Carbon/Carbon Nanotube) Electrode Materials for Supercapacitor: A review. J. Inorg. Organomet. Polym. Mater. 2018, 28, 559-584. [CrossRef]

40. Chen, Y.; Zhang, X.; Zhang, H.T.; Sun, X.Z.; Zhang, D.C.; Ma, Y.W. High-Performance Supercapacitors Based on a Graphene-Activated Carbon Composite Prepared by Chemical Activation. RSC Adv. 2012, 2, 7747-7753. [CrossRef]

41. Ma, W.; Chen, S.; Yang, S.; Chen, W.; Wei, W.; Zhu, M. Bottom-Up Fabrication of Activated Carbon Fiber for All-Solid-State Supercapacitor with Excellent Electrochemical Performance. ACS Appl. Mater. Interfaces 2016, 8, 14622-14627. [CrossRef] [PubMed]

42. Xie, Q.; Zheng, A.; Zhai, S.; Wu, S.; Xie, C.; Zhang, Y.; Guan, Y. Reed Straw Derived Active Carbon/Graphene Hybrids as Sustainable High-Performance Electrodes for Advanced Supercapacitors. J. Solid State Electrochem. 2016, 20, 449-457. [CrossRef]

43. Zheng, C.; Zhou, X.F.; Cao, H.L.; Wang, G.H.; Liu, Z.P. Nitrogen-Doped Porous Graphene-Activated Carbon Composite Derived from “Bucky Gels” for Supercapacitors. RSC Adv. 2015, 5, 10739-10745. [CrossRef]

(C) 2018 by the authors. Licensee MDPI, Basel, Switzerland. This article is an open access article distributed under the terms and conditions of the Creative Commons Attribution (CC BY) license (http://creativecommons.org/licenses/by/4.0/). 